\title{
New paradigm for nuclear data evaluation
}

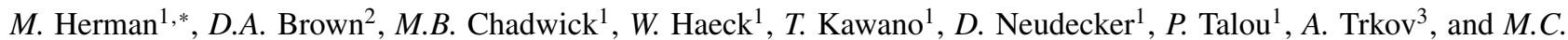 \\ White $^{1}$ \\ ${ }^{1}$ Los Alamos National Laboratory, Los Alamos, NM 87545, USA \\ ${ }^{2}$ Brookhaven National Laboratory, Upton, NY 11973-5000, USA \\ ${ }^{3}$ International Atomic Energy Agency, PO Box 100, A-1400 Vienna, Austria
}

\begin{abstract}
A new paradigm for nuclear reaction data evaluations is proposed to produce adjusted libraries that take into account integral experiments on the same footing as the differential ones. These evaluations will provide comprehensive covariance matrices including cross-correlations among different materials/reactions that are critical for realistic propagation of data uncertainties to integral quantities. The new approach should also reduce error compensation issues and facilitate updating of the library to account for new or corrected experiments and advances in reaction modeling.
\end{abstract}

\section{Introduction}

Building on the results of a series of NEA WPEC Subgroups 47, 46, 45, 40, 39, 33, 26 [1], IAEA-led International Nuclear Data Evaluation Network, experience gained from the American Recovery and Reinvestment Act projects, and recent progress in nuclear reaction modeling and machine learning, we propose a change in the evaluation procedure to produce adjusted neutron-induced reaction libraries which would consistently account for differential and integral experiments.

Most modern reaction evaluations consider all available differential data, after possible correction for e.g., updated values of reference cross sections, Neutron Data Standards, spectroscopic data, half-lives, etc. and use these data to calibrate model calculations to reproduce these measurements as far as possible. In certain cases, differential data are replacing model calculations, e.g., when strong cross-section fluctuations are observed but the bulk of such evaluated file is determined by reaction model calculations. Since such a file is not affected by the integral experiments we will call it in the following "pure differential". It is important to note that the reaction modeling is playing an important role by imposing physics constraints and providing a complete set of data for quantities and/or energy ranges where there are no measurements or available data are insufficient. This is a strength of the current evaluation procedure compared to curve fitting which can be applied only in very limited cases where experimental results are complete and abundant.

The "pure differential" libraries, however, perform inadequately in the integral testing because the precision of integral experiments is usually far better than the precision of differential measurements and far beyond the predictive

*e-mail: mwherman@lanl.gov power of "a priori" model calculations. To achieve the accuracy required by various applications, most of the current libraries, with notable exception for JENDL, make use of integral experiments to inform "pure differential" evaluations. This leads to a manual, sensitivity guided, tuning (or calibrating) of certain evaluated quantities, admittedly within differential uncertainties, in order to improve the overall performance of the library on a set of relevant integral measurements.

While the tuning often results in much better agreement with benchmarks, another strength of the current approach, it comes at a price. Usually, only central values of the data are changed, in some cases by modifying model parameters, but cross-reaction and cross-material correlations are not created, e.g., between $\bar{v}$ and fission. This leads to a situation where integral mean values are pretty well reproduced by transport calculations using tuned libraries but propagated library uncertainties are too large for fission applications, e.g., criticality experiments. The next side effect comes from the human nature; a manual tuning tends to pick up the most sensitive parameter and exploits it to the utmost outcome. This ignores other possible factors which, although individually less important, together could contribute to a significant effect.

The omission mentioned above may lead to overcorrection of the adjusted parameter and is one of the possible sources of the so called "error compensation". Similarly, an existing deficiency in an evaluation for some other material may induce unnecessary compensation adjustment in the currently evaluated file. These are exacerbated by the fact that only a limited number of materials is reevaluated in each release of the library. Therefore, a potential error in one of the preserved evaluations locks a compensating error in the newly evaluated material since correcting it without fixing its counterpart would degrade the library performance. 
So, while the "pure differential" evaluation procedure for a single material is sound and the tuned libraries perform quite well, they suffer from the following deficiencies:

- Integral experiments are often partially taken into account but this is not reflected in the evaluated covariances.

- The full validation is performed after the library has been released (or frozen) so its feedback can not be used until the next release, years later.

- There is no easy way to make even slight change to the evaluation of a material that is not currently being evaluated (e.g., to remove a compensating error).

- The documentation is not sufficient to reproduce the evaluation, thus each time it has to be redone from scratch.

The new evaluation paradigm outlined in this paper intends to address these issues.

\section{New paradigm}

The new paradigm consists of a few principal ideas that require a number of conditions to be fulfilled in order to work effectively and efficiently.

\subsection{Principal ideas}

The new paradigm retains the current "tuned library" and adds "pure differential" and "adjusted" libraries. Even though it seems to be compounding significant additional burden on evaluators and Data Centers it shouldn't be too bad since a "pure differential" file is always the first step in any evaluation. The other two libraries can simply branch out from the "pure differential" trunk in the version control system. Preserving the "pure differential" library, even though likely ineffective for specific applications, will be valuable for keeping track of the evaluation procedure and separating effects of tuning or adjusting from the data not affected by integral experiments. For the materials not present in integral measurements the "pure differential" files will be the only entries in the library.

The essential novelty of the proposed approach is the adjustment of the entire library when any new or changed evaluation is committed to the "pure differential" branch. This might happen on a daily basis and will require adequate arrangements that will be discussed later. It implies that any change in one evaluation may give rise to changes in other evaluations as a result of the adjustment procedure. This opens a possibility of reducing error compensation between evaluations A and B by automatically adjusting $\mathrm{B}$ (possibly also $\mathrm{C}, \mathrm{D}, \ldots$ ) to the change in A. One has to proceed with caution, however, since the same mechanism might also generate an error compensation if the change in $\mathrm{A}$ is unjustified. Because of the adjustment there will be three flavors of evaluation in the new library (i) newly evaluated and adjusted, (ii) modified by the adjustment, and (iii) migrated from the previous version without any change. Accounting for integral experiments will provide a comprehensive covariance matrix including cross-correlations among different materials/reactions that are critical for realistic propagation of data uncertainties to integral quantities.

On the organizational level the new paradigm would re-dimension the importance of the post-release validation by integrating major part of it into the evaluation (adjustment) procedure. This would necessitate the direct involvement of the validation community in the evaluation process bringing the two communities into close collaboration.

\subsection{What does the new paradigm imply?}

The global adjustment and validation performed on a nearly daily basis calls for a modern infrastructure in order to make the task feasible and to ensure a high quality for the produced libraries. The list below focuses on the substance and intentionally avoids technical details (responsible communities are indicated within brackets):

1. Storing all the details of evaluations in an electronic form (inputs, codes, experimental data, corrections, assembly scripts, and sensitivity profiles) to the extent that enables reproducing the evaluation and its automatic adjustment or, if needed, re-evaluation within days (evaluation community).

2. Establishing a set of clean, reliable and representative integral experiments, along with the respective decks, and sensitivity profiles, covering the whole available application field to be used in the adjustment. This set should be balanced between different types of integral experiments and various types of applications. It should also be free of outliers and measurements with unreasonably small uncertainties (validation community).

3. Developing the capabilities that enable quick adjustments of the relevant parts of the library to the changes in the evaluation for a given material (it might be beneficial to employ more than one adjustment method) (validation, evaluation and machine learning communities).

4. Developing an automated adjustment-review process which will allow evaluators to judge the quality and acceptability of the adjustment. If any adjustment exceeds a pre-set upper limit, it should be individually reviewed and, eventually, the material should be reevaluated (validation, evaluation and machine learning communities).

5. Setting up a repository for storing and versioning all items mentioned in the points above (international organization and/or evaluation projects).

The first point will probably be the most time and work intensive part of the project as practically no existing evaluation fulfills such extended documentation requirement. This condition has to be imposed on all new evaluations while old evaluations of primary importance could 
be "cloned", i.e., reproduced within desired accuracy by model calculations that is far easier and faster than a full scope new evaluation.

An implicit ingredient essential to this procedure is the further development of reaction models to ensure that all relevant physics mechanisms are properly represented, and the models are appropriately parametrized. Judicious transition to more microscopic models would have a decisive impact on the overall quality of the library.

\subsection{What are the advantages of the new paradigm?}

The new paradigm requires substantial reorganization of the evaluation procedure and substantial investment before the new blueprint can be put into operation. It is, therefore, wise to layout some of the benefits that justify such an effort:

- Introduction of cross-correlations among nuclear data imposed by the inclusion of integral measurements in the adjustment. These will reduce uncertainties on the integral quantities calculated by propagating uncertainties of the basic nuclear data making nuclear data covariances more sensible to the users.

- Knowledge of the cross-correlations will point to possible compensation of errors facilitating their removal.

- Automatic adjusting of evaluations for all materials will release evaluators from their being unable to remove an error in the file being evaluated because it compensates an error in another material(s).

- Introduction of new experiments or model developments will be much easier since the whole system will be set up to rerun each evaluation with minimal effort and to re-adjust the whole library to this change.

- New evaluations will be assisted by the availability of extensive details of previous evaluations that will largely simplify and speed up new evaluations of the material (in some cases simple Bayesian update might be sufficient). Recorded evaluation history will be a key factor in know-how preservation and an excellent material to train new evaluators. It will also decrease the probability of overlooking certain aspects of the evaluation procedure or particularities specific to a given material.

- A database of validation results, accumulated in the process of frequent adjustments with atomic changes to the nuclear data libraries, will provide a rich source for investigation and excellent learning set for artificial intelligence algorithms, especially if meta-data for the experiments and reaction model inputs are stored.

- Close interaction of the evaluation and validation communities will result in a better, more focused, and more reliable reaction data library.

\subsection{What should be the adjustment strategy?}

The details of the adjustment strategy will be a subject of thorough debate and personal preferences. We do not want to get into these deliberations now, as this paper is focused on the overall idea rather than technical details. It is obvious, however, that the choice of the adjustment method as well as of the adjustment strategy will have a decisive impact on the success or failure of this paradigm. Below we outline one of possible scenarios of the adjustment strategy to illustrate the difference between a strategy and a method (e.g., least-square fitting, Kalman filter, simulated annealing, or machine learning techniques).

An example strategy that we put forward here is a gradual (sequential) assimilation approach. The assimilation, or consistent adjustment, makes use of differential and integral data to determine reaction model parameters. The actual evaluation is produced by a Reich-Moore [2] approximation to the R-matrix [3] in the resolved resonance region, and by the reaction model calculations in the fast neutron range. In both cases, the parameters are searched to reproduce as far as possible the whole set of experimental data (see Sec. 3.3 for more details). The advantage of this approach is that all physics constraints are observed and the predictive power of the model is exploited to interpolate and extrapolate to experimentally unexplored quantities and energy regions.

Figure 1 illustrates the assimilation of ${ }^{239} \mathrm{Pu}$ cross sections and Prompt Fission Neutron Spectra (PFNS) to the Jezebel benchmark performed within the exploratory work on the assimilation methodology [4] carried out by a collaboration between Brookhaven and Idaho National Laboratories. In this exercise 53 model parameters were varied out of which 22 were modified. These covered level densities, fission and PFNS, but the most prominent player $\bar{v}$ was not included, since it is not modeled by the EMPIRE code [5]. This implies that the potential changes in $\bar{v}$ were absorbed by the fission cross sections, because of the well known correlation between the two quantities. The average change calculated for the 22 parameters was only $0.02 \%$. The maximum modification was for the hight of the second-hump in ${ }^{240} \mathrm{Pu}$ fission barrier, which was increased by $0.42 \%$. Figure 1 only shows the results for the fission cross sections since changes in other channels are even smaller. This proof of concept exercise demonstrates the feasibility of the assimilation procedure. It dramatically improved agreement with integral experiment and led to the reduction of uncertainties in the model parameters and consequently also in the calculated integral result. The major correlations are shown and discussed in Ref. [4] but missing $\bar{v}$ eliminates the most important factor. Interestingly, the Jezebel benchmark practically does not correlate the PFNS parameters with the remaining ones.

Let us now explain the meaning and importance of the gradual approach. Typical differential experiments provide results for a well defined nuclear reaction on a specific nucleus in a given, usually narrow, energy interval. On the other hand, complex integral measurements average over incident energies, various reactions and various materials to which we often need to add a complicated geometry. Therefore, involved transport calculations are needed to link basic nuclear data with the results of integral experiments. Generally, with differential experiments we know exactly what is being measured but the precision is moderate (except for a few special cases no better 


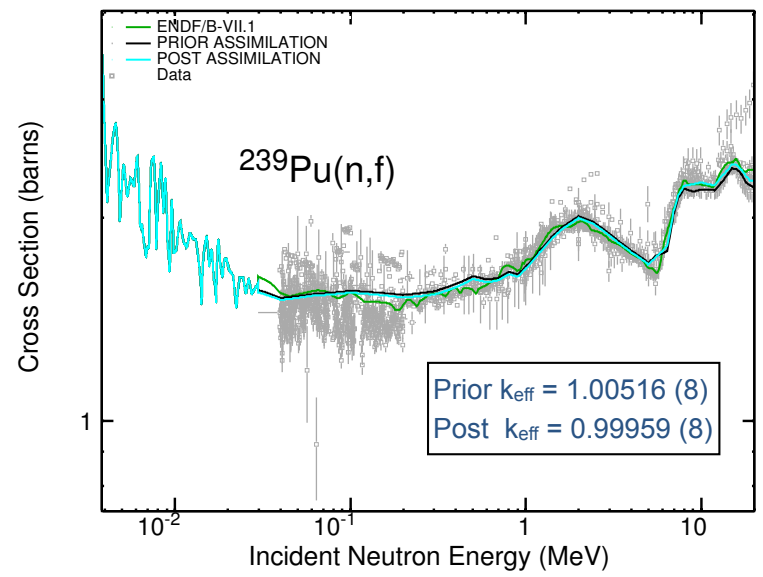

Figure 1. Assimilation of ${ }^{239} \mathrm{Pu}$ cross sections to Jezebel integral measurement showing how minimal cross section changes are needed to bring over-prediction of $k_{\text {eff }}$ by 516 pcm down to -41 pcm.

than $2 \%$ ). On the contrary, the precision of integral experiments is generally much higher (a fraction of a percent for criticality), but we are not able to disentangle individual nuclear data components. Like in the Heisenberg principle, if we know what we are measuring, we know the result with limited accuracy. If we do measure with a high accuracy, we do not really know what we measure (in terms of nuclear data quantities). This feature gives grounds for the gradual adjustment approach. First, we take advantage of the "most selective" experiments, which directly constrain a subset of model parameters. Then, we move progressively to less selective differential experiments followed by semi-integral experiments ending up with integral ones of increasing complexity. At each step we should produce covariances to be used in the next round of adjustment to ensure that assimilation of integral measurements does not perturb model parameters, determined previously by the specifically sensitive experiments, by more than a prefixed fraction of their uncertainty.

Figure 2 outlines the procedure for a new evaluation. As mentioned above, experimental results for total, elastic, inelastic and averaged resonance quantities are used to determine parameters of the optical model which depend uniquely on these experiments. Other differential experiments are less selective in determining the remaining model parameters. At this stage we are in a position to produce a "pure differential" file using well established evaluation techniques, e.g., Bayesian approach. This file can be validated or modified in the Adjustment 1 phase which typically involves experiments informing the evaluation about specific reactions on a single material but integrate over the energy. This adjustment stage might still be considered to be part of the differential evaluation since the measured energy integral can be used to normalize model predictions without affecting energy dependence. In addition, there is usually no risk of introducing compensating errors. The remaining three stages of the adjustment are performed on the sensitive part of the library and can lead to changes in other materials, which is the essential novelty of the proposed paradigm. According to the gradual approach, we start with the integral experiments involving single material and move to simple geometry experiments containing a few materials to complete with moderately complex setups.

One of the fundamental assumption of the new paradigm is that the changes resulting from adjustments in the last three stages are small in comparison to the uncertainties of the experimental cross sections (see Fig. 1). Consequently, also changes in the model parameters are expected to be tiny when compared to the physics constraints on the parameters. This assumption stems from the fact that current libraries, which are supposed to be a starting point for the new paradigm, perform quite well in the validation. The evaluations causing major discrepancies in comparison with integral experiments have already been fixed. If changes suggested by the adjustment procedure are larger than a specified limit (e.g., one standard deviation) the offending case should be carefully reviewed. It might turn out that certain benchmarks need to be revised or the evaluation should be redone.

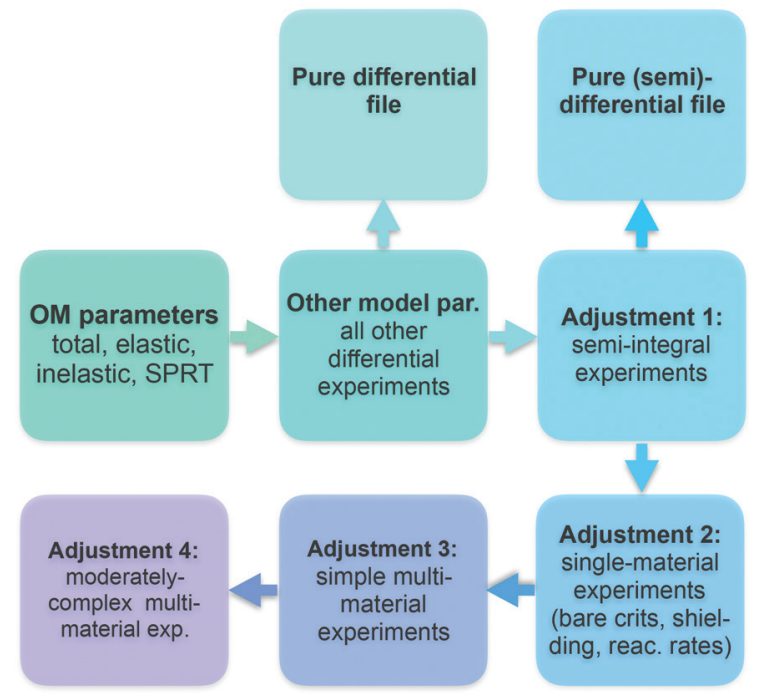

Figure 2. An example of a possible evaluation + adjustment scenario based on the gradual inclusion of less and less specific (i.e., more integral) experiments. The two upper rows represent a new evaluation for a single material which results in a puredifferential (or semi-differential) file. The bottom row represents the three steps of adjustment that involve all materials/reactions which are significantly contributing to the integral experiments sensitive to the new evaluation.

\subsection{Is there a role for machine learning?}

Recently, machine learning techniques have been vigorously developed and scored remarkable successes in many fields. Their application to nuclear data started a couple of years ago and the first results are encouraging $[6,7]$. It is too early to predict the extent to which machine learning will be employed in the proposed paradigm, but some 
expectations can be outlined. At the minimum, machine learning an be used to automate and greatly speed up the analysis of each adjustment which might be needed on a daily basis. Next, machine learning may help discover hidden correlations, identify outliers, point to possible error cancellations, and eventually even perform the adjustment. Responsiveness of the analysis and the capability of dealing with big data will be the decisive factors as we do not have the manpower to promptly analyze results of the adjustment on a very irregular time schedule.

\subsection{Why is it time to make a change?}

The primary argument for adopting the new paradigm is the fact that our libraries perform pretty well. Hence they are probably pretty close to the truth, and even linear adjustment has a good chance to be effective. Then, discrepancies among integral measurements and simulations are distributed among the components of nuclear data libraries, making manual tuning impractical. Improving the overall performance will be more and more difficult unless we upgrade our approach.

Also, including integral experiments becomes easier to accept since current differential experiments depend more and more on transport calculations to account for various corrections. Thus the difference between differential and integral experiments is already blurred to some extent.

Finally, we are in a much better position to implement the new paradigm today than 20 years ago because of: (i) available computer power, (ii) improvements in reaction modeling, (iii) availability of benchmarks, (iv) availability of sensitivity profiles and related infrastructure, (v) new measurements with smaller uncertainties (Chi-Nu [8], TPC [9], LENZ [10], ...), (vi) progress in adjustment methods (WPEC Subgroups), (vii) availability of the 'Advance System' at NNDC, and last but not least (viii) ascent of machine learning techniques.

\subsection{What else could be done?}

There is a tendency to include reaction modeling inside the transport codes. This implies abandoning, at least partially, the traditional tabulated (formatted) libraries in favor of performing reaction calculations alongside Monte Carlo transport calculations. This approach requires substantial increase in the computational power but has an advantage of making use of all the details provided by the reaction codes (e.g., CGMF [11]), such as various correlations, typically present in a reaction model code, that cannot be included in the ENDF file. As far as the basic concept of assimilation is concerned this approach does not differ from the assimilation scenario described in Sec. 2.4 since in both cases the model parameters are those being adjusted. Also, going beyond linear approximation (covariances) is natural within this scenario.

\section{Concerns}

In this section, we address concerns that might be raised regarding the new paradigm. They are of fundamental im- portance and their discussion gives more insight into the proposed approach.

\subsection{Will including integral experiments bias the library?}

Including integral experiments in the general purpose library has been vigorously disputed over decades. Though the needle has been gradually moving from total negation towards a caution acceptance there is still no consensus regarding the idea even among the authors of this paper. Therefore we propose to maintain three types of libraries the "differential", the "tuned", and the "adjusted" and leave the final decision until we can compare the performance of the latter two.

Meanwhile, we give a few arguments why the "adjusted" library might not be biased by integral experiments more than any "tuned" one. Covering all available integral experiments in the representative mode and eliminating those experiments that are deemed not reliable, we should be in a position to obtain more general results than the previous, application specific, adjustments. We also expect rather small changes in the nuclear data, at least for those accepted in the automated global readjustment phase(s), since larger modifications will call for evaluators' and validators' analysis and potential reevaluation. The use of the reaction models in the adjustment will eliminate a risk of creating unphysical structures (fluctuations) in the data, which is always possible if physics unconstrained adjustment is performed. So we expect the adjusted file to be similar to the "tuned" one but with the changes distributed over many nuclear data quantities instead of affecting one or two. The broader distribution will make changes smaller, and therefore even more acceptable.

\subsection{Are (cross-)correlation covariance matrix elements real?}

Covariances are a key ingredient in any linear adjustment, since they directly govern the whole process and define the result. It is therefore of utmost importance to ensure that they are credible. Actually, to preserve differential experiment constraints in the adjustment procedure, it might be desirable to maintain tight uncertainties on the "pure" files.

Including experimental constraints always introduces correlations. In the case of differential experiments these are energy self-correlations and cross-reaction correlations that are imposed by the reaction physics or details of the experimental procedure. The correlations among differential experiments, being difficult to establish, are rarely considered. Even if they were, in most cases, they would probably be overwhelmed by the correlations imposed by the physics.

The integral experiments impose cross-reaction and cross-material correlations which come on top of those introduced by the differential experiments. Since uncertainties of integral experiments are usually much lower than 
those of differential ones these cross-correlations are instrumental in bringing down uncertainties of integral experiment simulations obtained by propagating nuclear data covariances. This is one of the main advantages of the new paradigm.

It is often questioned whether such correlations are real. In general, these covariances do not account for the possible correlations among various experiments. So, one of the most intuitive sources of correlations is missing, although it could be included if pertinent information about experiments were available. The correlations, however, account for the fact that available experimental information can be interpreted via different sets of nuclear data and, therefore, different sets of model parameters. In the case of differential experiments, these correlations reflect the underlying reaction physics, while in the case of integral experiments, they reflect the design of a benchmark and the transport theory.

Uncertainties and their correlations are not physical quantities but quantification of our knowledge limits. As such, they are time and situation dependent. The same way the cross section uncertainties depend on the differential experiments included in the evaluation, the cross-material correlations depend on the integral experiments included in the adjustment. Similarly to a new, very precise, cross section measurement reducing a cross section uncertainty, a new very precise integral experiment will increase crossmaterial or cross-reaction correlations (it might also lead to the decrease of the uncertainty). It is unreasonable to expect universal, constant in time covariances. It is, however, reasonable to expect that covariance matrices built independently by two groups of evaluators, out of the same data, will be similar.

\subsection{Can we trust reaction models?}

With "reaction model" we mean here anything between phenomenological and first-principle microscopic approaches, i.e., the current best evaluation practice, which will naturally evolve in time. The whole energy range has to be divided in at least two regions, the resonance region and the fast neutron region.

The resonance region benefits from the exact R-matrix theory, or various simplifications of it, with Reich-Moore being the most common. While the physics foundation of the R-matrix is sound it still requires experimental data since the R-matrix approach is not able to make a-priori predictions. The fitting procedure transforms these experimental data into model (resonance) parameters that together with the R-matrix formulation define the cross sections and scattering angular distributions in the region. We know exactly what are the limits of ENDF-6 approximations to R-matrix and we can fully trust our resonance model. This leaves us only with the ambiguity regarding resonance parameters. We can vary them within experimental uncertainties and this approach has already been explored [12]. The essential problem here is that for actinides we have far more parameters than integral constraints and an adjustment has to be more selective. Also the possibility of loosing resonances should be taken into account adding additional degrees of freedom in the highenergy end of the resonance region.

In the fast neutron region, cross-sections, spectra, angular distributions and double differential cross-sections can be reproduced within experimental uncertainties using a combination of the optical model, coupled-channels model, preequilibrium and statistical models (including fission). For more fine tuning, or to account for possible model defects, we can use energy-dependent correction factors that allow to adjust cross sections without violating any physical constraints. Figure 3 shows overall good agreement between EMPIRE [5] calculations using adjusted parameters and standards cross sections for the ${ }^{235} \mathrm{U}(\mathrm{n}, \mathrm{f})$ cross sections. There is, however, a structure between 0.3 and $1 \mathrm{MeV}$ that can not be reproduced by varying model parameters. If this structure is real (and we generally trust the standards), we must admit that the model is deficient in this region. This deficiency may be corrected by invoking an energy-dependent tuning of the fission width. Figure 3 shows that the observed structure can be reproduced without compromising conservation laws imposed by reaction physics. Using corrective factors acting on various internal components of the reaction models we may reproduce any reaction cross section with an arbitrary precision without resorting to least-square fitting, splines, or eye-guided lines.

The new paradigm does not require a full-fledged physics model for all physical quantities but, if the model is lacking, it needs a suitable parametrization to enable adjustment. Our $\bar{v}$ and PFNS modeling has not yet reached the same level of confidence as calculations of reaction cross sections although progress is being made $[11,13$, 14]. While having a sound physical modeling is always preferred, a suitable parametrization (e.g., the Los Alamos model for PFNS [15]) is an acceptable solution. The paradigm is designed to facilitate the implementation of the modeling improvements when they are ready for deployment.

\subsection{What if different adjustment methods produce different results?}

Different adjustments tend to produce somewhat different results. This is the reality we have to face. While it is unfortunate it is nothing new. Different experiments and different reaction codes often produce results which are inconsistent, even evaluations contradict each other. It is up to the evaluation community to sort out the discrepancies. It is likely that we will encounter a similar problem in adjustment but we will be in a position to count on the support of the validation community. Actually, it might be beneficial to use several adjustment methods and strategies to gain more insight into the nature of adjustment and to gain more confidence in the results. For example, the classical adjustment (i.e., without involving reaction modeling), being more flexible, might point to particular energy intervals or suggest some structures (there will be a lot to discuss during the annual meetings). 


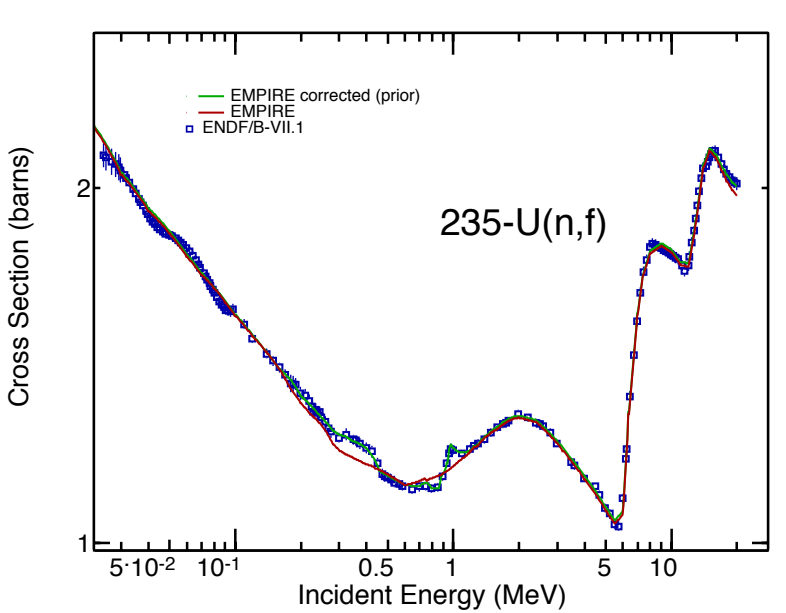

Figure 3. Reproduction of the fission cross section on ${ }^{235} \mathrm{U}$ with the EMPIRE code calculations (red solid line). Model parameters were adjusted to fit ENDF/B- VII.1 data coming from the standards. In two energy regions it was not possible to reproduce standard's cross sections with pure model calculations and energy dependent parameters had to be invoked (green solid line). This example illustrates that one can take care of "model defects" using energy dependent model parameters.

\section{Conclusions}

We propose a more integrated and global approach to developing nuclear reaction data libraries. The fundamental evaluation procedure remains very much the same, but the assembly of the library involves global adjustment to the comprehensive set of selected, representative and trustworthy integral experiments. This adjustment is performed each time a new or updated evaluation is submitted and potentially modifies other evaluations in the library. Consequently, the validation community becomes an integral part of the evaluation team. Each adjustment undergoes a rigorous scrutiny aided by the dedicated software and machine learning techniques. If changes exceed certain limits they have to be reviewed before being accepted. In general, however, we expect these changes to be small.

In order to implement this paradigm we need to preserve an explicit (digital) memory of each evaluation to allow for a quick adjustment. This will also facilitate potential reevaluations in the future. We also need to develop the software infrastructure for datamining within databases of integral experiments, sensitivity profiles, adjustment results, and other quantities of interest. These retrieval, analysis and visualization tools will be essential since the amount of information to be digested might be overwhelming.

In addition to the above mentioned "adjusted" library we propose to maintain the current "tuned" library and add a "differential" library that is not affected by integral experiments. The latter will allow to separate effects of the adjustment and will facilitate reevaluation.

The scope of the proposed paradigm might seem intimidating but actually many of the proposed activities are being carried out already. Four out of five requirements listed in Sec. 2.2 are, indeed, under development:

1. reproducibility of the evaluations is being addressed by WPEC SG 49 [16],

2. WPEC SGs 45 [17] and 47 [18] are working on the set of recommended integral experiments (with SG 47 dealing with shielding benchmarks),

3. adjustment methodology has been the subject of numerous WPEC Subgroups (46 [19], 39 [20], 33 [21, 22], 26 [23]), while the automated validation system is under development at NNDC and related infrastructure is currently being developed at LANL and NEA,

4. -

5. the repositories are already active at NNDC and NEA.

The missing point 4 - developing automated adjustment-review system will be facilitated by modern tools and machine learning techniques. In addition, the pilot project on usage of machine learning techniques in nuclear data is underway at LANL $[6,7]$. The aim of the new paradigm is to integrate all these activities, bring closer various communities, and assimilate all experimental information and nuclear reaction theory to provide the best currently possible nuclear reaction libraries and facilitate their future improvements.

The new paradigm is scalable and can be started with a limited core of nuclei, e.g., those considered in the CIELO [24] exercise plus a few other most important nuclei, so that its viability may be tested within a relatively limited effort.

This work was performed under the auspices of the National Nuclear Security Administration of the U.S. Department of Energy at Los Alamos National Laboratory under Contract No. 89233218CNA000001, LA-UR-19-28926.

\section{References}

[1] Working Party on International Nuclear Data Evaluation Co-operation (WPEC), https://www.oecdnea.org/science/wpec/

[2] C. W. Reich and M. S. Moore, "Multilevel Formula for the Fission Process", Phys. Rev. 111929 (1958).

[3] A. M. Lane and R. G. Thomas, "R-matrix theory of nuclear reactions", Rev. Mod. Phys. 30257 (1958).

[4] M. Herman, S. Hoblit, G. P. A. Nobre, A. Palumbo, M. T. Pigni, G. Palmiotti, H. Hiruta, M. Salvatores, "Use of Covariances in a Consistent Data Assimilation for Improvement of Basic Nuclear Parameters in Nuclear Reactor Applications: From Meters to Femtometers", BNL Report BNL-99142-2013-IR, https://www.bnl.gov/isd/documents/79809.pdf

[5] M. Herman, R. Capote, B. Carlson, P. Obložinský, M. Sin, A. Trkov, W. H., and V. Zerkin, "EMPIRE: Nuclear Reaction Model Code System for Data Evaluation," Nuclear Data SheEts, 108, 2655 (2007). 
[6] P. Grechanuk, M. E. Rising and T. S. Palmer, "Using Machine Learning Methods to Predict Bias in Nuclear Criticality Safety", Jour. of Computational and Theoretical Transport, 47, NOS. 4-6, 552 (2018), https://doi.org./10.1080/23324309.2019.1585877

[7] P. Grechanuk, M. E. Rising and T. S. Palmer, "Identifying Sources of Bias from Nuclear Data in MCNP6 Calculations using Machine Learning Algorithms", M\&C 2019, International Conference on Mathematics and Computational Methods Applied to Nuclear Science and Engineering, 2019-08-25, Portland, Oregon, United States.

[8] https://lansce.lanl.gov/facilities/wnr/flight-paths/chinu/index.php

[9] https://lansce.lanl.gov/facilities/wnr/flight-paths/fp15R/index.php

[10] https://lansce.lanl.gov/facilities/wnr/flight-paths/fp90L/index.php

[11] B. Becker, P. Talou, T. Kawano et al., " Monte Carlo Hauser-Feshbach Predictions of Prompt Fission $\gamma$ Rays: Application to $n_{\mathrm{th}}+{ }^{235} \mathrm{U}, n_{\mathrm{th}}+{ }^{239} \mathrm{Pu}$, and ${ }^{252} \mathrm{Cf}$ (sf)", Phys. Rev. C 87, 014617 (2013).

[12] L. Leal and R. Sayer, "CSEWG-2014: Resonance Evaluations for ${ }^{16} \mathrm{O}$ for the CIELO Project."

[13] R. Vogt, J. Randrup, J. Pruet et al. , "Event-by-event Study of Prompt Neutrons from ${ }^{239} \mathrm{Pu}(n, f)$," Phys. Rev. C 80, 044611 (2009).

[14] O. Litaize and O. Serot, "Investigation of Phenomenological Models for the Monte Carlo Simulation of the Prompt Fission Neutron and $\gamma$ Emission," PHys. Rev.C 82, 054616 (2010).

[15] D. G. Madland and J. R. Nix, "New Calculation of Prompt Fission Neutron-Spectra and Average Prompt Neutron Multiplicities," Nucl. ScI. AND ENG., 81, 213271 (1982).

[16] D. Rochman and M. Herman, "WPEC-SG49 Reproducibility in Nuclear Data Evaluation," https://www.oecd-nea.org/science/wpec/sg49/
[17] M. White and D. Bernard, "WPEC-SG-45 Validation of Nuclear Data Libraries (VaNDaL) Project," https://www.oecd-nea.org/science/wpec/sg45/

[18] I. Kodeli, "WPEC-SG-47 Use of Shielding Integral Benchmark Archive and Database for Nuclear Data Validation," https://www.oecdnea.org/science/wpec/sg47/index.html

[19] M. Salvatores and G. Palmiotti, "WPEC-SG46 Efficient and Effective Use of Integral Experiments for Nuclear Data Validation.," https://www.oecdnea.org/science/wpec/sg46/

[20] G. Palmiotti and M. Salvatores, "WPEC-SG-39 Methods and Approaches to Provide Feedback from Nuclear and Covariance Data Adjustment for Improvement of Nuclear Data Files," https://www.oecdnea.org/science/wpec/sg39/

[21] G. Palmiotti and M. Salvatores, "WPEC-SG-33 Methods and Issues for the Combined Use of Integral Experiments and Covariance Data," https://www.oecdnea.org/science/wpec/sg33/

[22] M. Salvatores, G. Palmiotti, G. A. Aliberti et al., "Methods and Issues for the Combined Use of Integral Experiments and Covariance Data" A report by the Working Party on International Nuclear Data Evaluation Co-operation of the NEA Nuclear Science Committee NEA/NSC/WPEC/DOC(2013)445, Nuclear Energy Agency (2013).

[23] M. Salvatores, G. Aliberti, M. D. Dunn et al., "WPEC-SG-26 Uncertainty and Target Accuracy Assessment for Innovative Systems Using Recent Covariance Data Evaluations," International Evaluation Cooperation 26 ISBN 978-92-64-99053-1, OECD Nuclear Energy Agency (2008).

[24] M.B.Chadwick, R.Capote, A. Trkov et al. , "CIELO Collaboration Summary Results: International Evaluations of Neutron Reactions on Uranium, Plutonium, Iron, Oxygen and Hydrogen", Nuclear Data ShEETs, 148, 189-213 (2018). 